\title{
Ischemic Stroke as the Initial Manifestation of Neurosyphilis in a Young Adult Patient Positive for Human Immunodeficiency Virus
}

\author{
Ayaka Numao ${ }^{a}$ Keisuke Suzuki ${ }^{a}$, Hidehiro Takekawa ${ }^{a}$, Toshiki Nakamura ${ }^{b}$, Hisatake Iwanami ${ }^{a}$, Naoki \\ Izawa ${ }^{a}$, Akio Iwasaki ${ }^{a}$, Hiroaki Fujita ${ }^{a}$, Yuji Watanabe, ${ }^{a}$ Koichi Hirata ${ }^{a}$ \\ ${ }^{a}$ Department of Neurology, Dokkyo Medical University, Tochigi, Japan \\ ${ }^{\mathrm{b}}$ Department of Neurology, Rehabilitation Amakusa Hospital, Saitama, Japan
}

\begin{abstract}
:
A 31-year-old man with pontine infarction was referred to our hospital for further evaluation and treatment. At admission, his neurological examination was unremarkable. No lymphadenopathy or skin lesions were found. The Treponema pallidum haemagglutination test, rapid plasma regain test and fluorescent treponemal antibody absorption test of immunoglobulin $\mathrm{G}$ were positive in both serum and cerebrospinal fluid (CSF). CSF analysis showed lymphocytic pleocytosis. The patient had male-to-male sexual contact and was found to be HIV positive. Physicians should be aware that acute ischaemic stroke may be the first manifestation of neurosyphilis in a young adult, especially with HIV infection.
\end{abstract}

Keywords: Neurosyphilis, human immunodeficiency virus, young adult, initial symptom

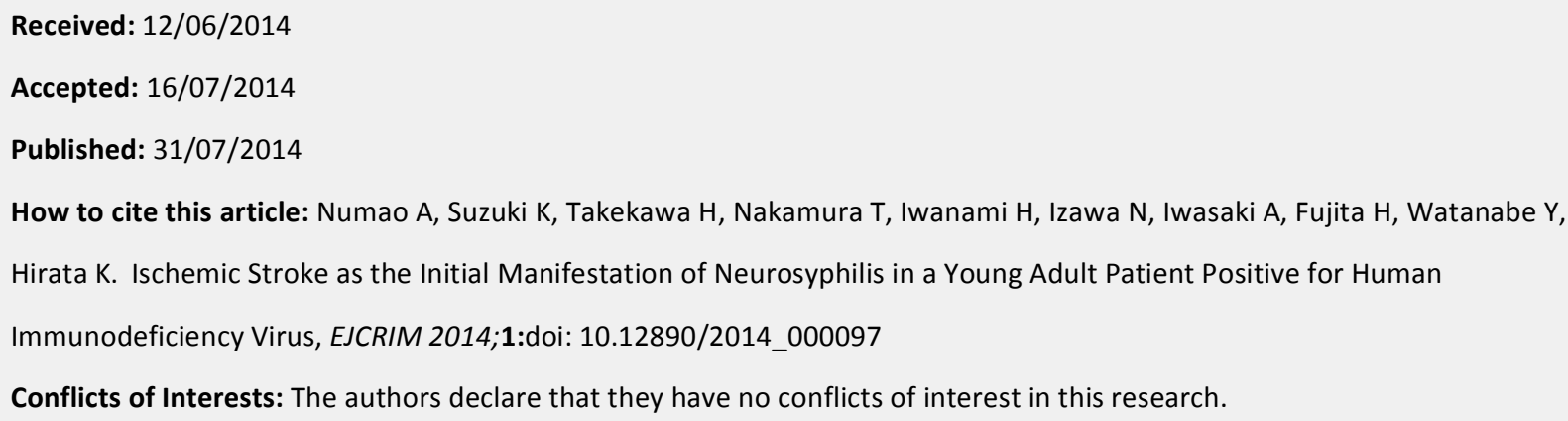




\section{Introduction}

The incidence of syphilis was reported to be 4.5 cases per 100,000 population in 2008 in the United States[1]. Recently the incidence of syphilis has been increasing, particularly among human immunodeficiency virus (HIV)-positive patients, with the prevalence of neurosyphilis being $0.4 \%$ in HIV-infected patients and $23.5 \%$ in HIV-infected patients with untreated syphilis[2]. In addition, the neurological manifestations in syphilis have changed since the beginning of the HIV epidemic. Central nervous system involvement including stroke can occur more frequently in the early stage in HIV-positive patients with syphilis than in HIV-negative patients with syphilis[3]. These findings suggest that HIV infection makes it difficult for physicians to correctly diagnose syphilis.

We here describe the case of an HIV-positive patient presenting with ischaemic stroke as the initial manifestation of meningovascular neurosyphilis.

\section{Case report}

A 31-year-old man developed acute left occipital pain which continued for 3 days. Then, 10 days later he developed left facial pain which lasted for 3 days followed by acute onset of dysarthria and right hemiparesis. Brain magnetic resonance imaging (MRI) performed at the previous hospital 19 days after the initial symptoms, showed acute brainstem infarction and so antiplatelet therapy with aspirin $100 \mathrm{mg} /$ day and cilostazol $200 \mathrm{mg} /$ day was started. The patient was referred to our hospital for further evaluation and treatment (Fig. 1).

\section{Left occipital pain}

Left facial pain

Dysarthria and right hemiparesis

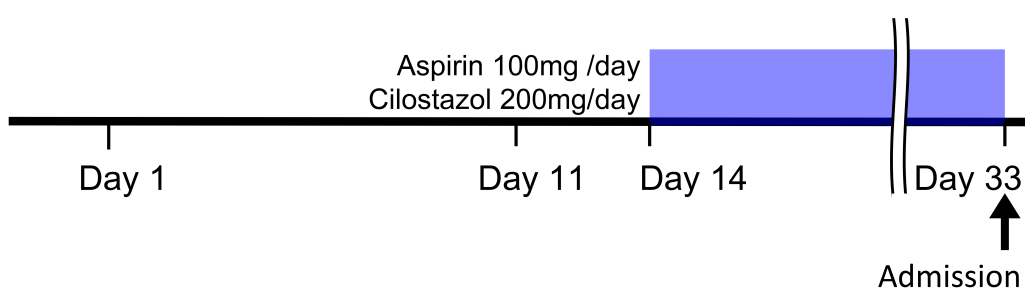

Figure 1: The patient's clinical course

He occasionally drank but did not smoke. His past medical history was unremarkable. At admission, his blood pressure was $124 / 74 \mathrm{mmHg}$, pulse was regular at $110 / \mathrm{min}$ and body temperature was $37.1^{\circ} \mathrm{C}$. Neurological examination was unremarkable. Brain MRI on fluid attenuated inversion 
recovery showed high signal intensities in the left pons (Fig. 2a) and periventricular white matter (Fig. 2b). Diffusion-weighted images showed no abnormality.

MR angiography showed irregular stenosis in the basilar artery and the proximal M1 segment of the middle cerebral artery (Fig. $2 c$ ).
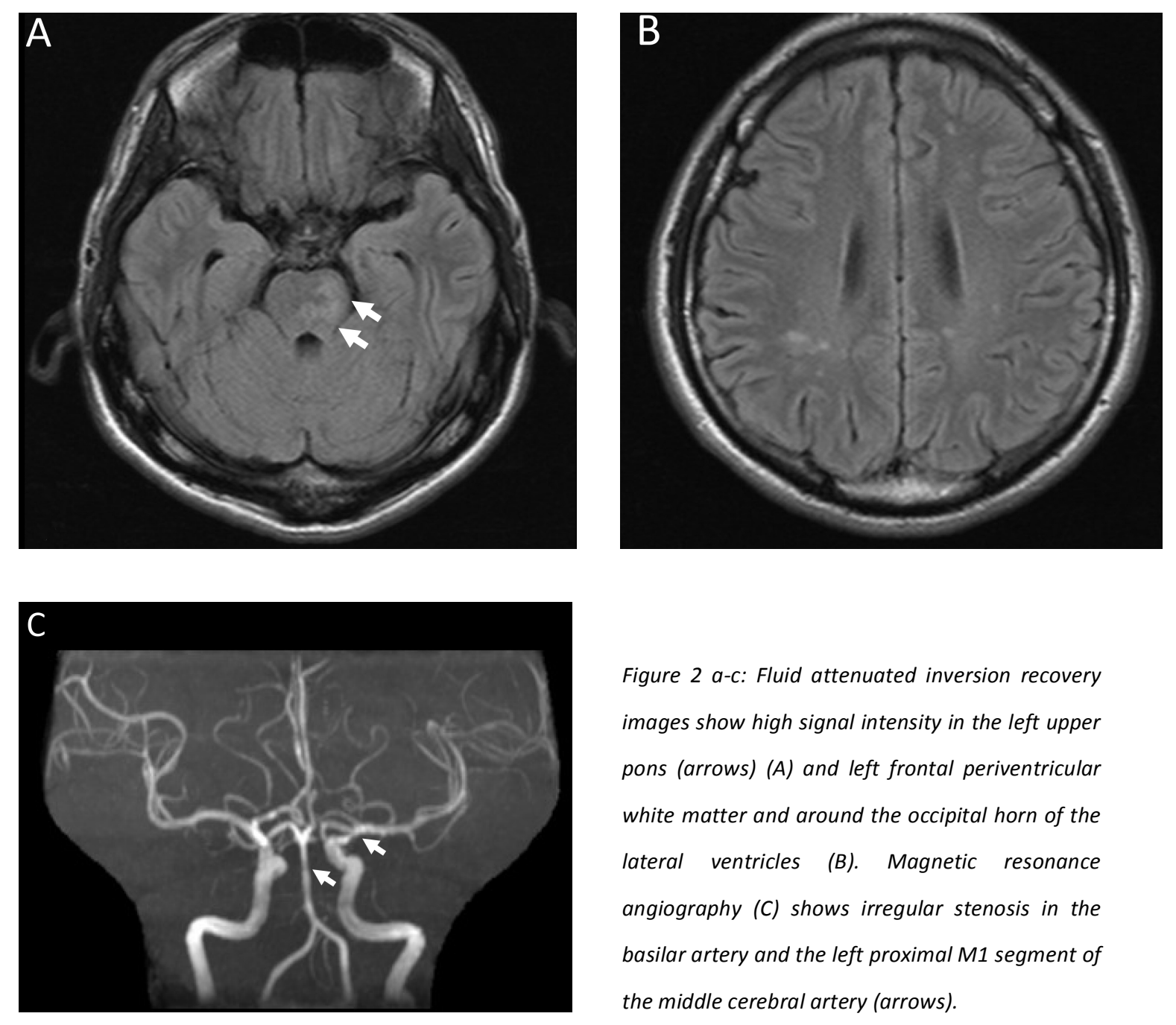

Figure 2 a-c: Fluid attenuated inversion recovery images show high signal intensity in the left upper pons (arrows) (A) and left frontal periventricular white matter and around the occipital horn of the lateral ventricles (B). Magnetic resonance angiography (C) shows irregular stenosis in the basilar artery and the left proximal M1 segment of the middle cerebral artery (arrows).

Electrocardiography (ECG) showed sinus rhythm, and continuous ECG monitoring did not detect paroxysmal atrial fibrillation. Cardiac sonographic findings were normal. Carotid artery sonography revealed normal flow patterns and no plaque in the common and internal carotid arteries. Funduscopic examination showed vitreous opacity and retinitis in the right eye. Laboratory data showed an elevated erythrocyte sedimentation rate $(40 \mathrm{~mm} / \mathrm{h})$, but C-reactive protein levels were normal. D-dimer levels were slightly increased at $0.7 \mu \mathrm{g} / \mathrm{ml}$. In both serum and cerebrospinal fluid (CSF), the Treponema pallidum haemagglutination (TPHA) test (serum, 16630 T.U.; CSF, $1569 \mathrm{U} / \mathrm{ml}$ ) and rapid plasma regain (RPR) test (serum, 1760 R.U.; CSF, 60.9 R.U.) and fluorescent treponemal antibody absorption test of immunoglobulin G (FTA-ABS) (serum, 1:320; CSF, 1:80) were positive. 
CSF analysis showed lymphocytic pleocytosis $\left(451 / \mathrm{mm}^{3}\right)$ with protein of $146 \mathrm{mg} / \mathrm{dl}$. The patient was found to be HIV positive with a CD4 count of 417 cells/ $\mu$ land plasma HIV RNA viral loads of 51,000 copies $/ \mathrm{ml}$. The risk factor for HIV infection in our patient was male-to-male sexual contact. The patient was treated with intravenous penicillin G (24 million units per day) for 2 weeks. By 17 days after the treatment, the CSF cell count and protein had decreased to $25 \mathrm{cells} / \mu \mathrm{l}$ and $126 \mathrm{mg} / \mathrm{dl}$, respectively. Highly active antiretroviral therapy was not started for HIV infection. After discharge, the CD4 count was monitored every 6 months. Six months later, the CSF cell count and protein, and FTA-ABS test (CSF) had significantly decreased to 12 cells $/ \mu \mathrm{l}, 78 \mathrm{mg} / \mathrm{dl}$ and 1:20, respectively. At the 21-month follow-up, the serum TPHA and RPR tests had improved (978 T.U. and 80.0 R.U., respectively). MR angiographic findings had not changed at the 2-year follow-up.

\section{Discussion}

We report the case of a young patient with concomitant HIV infection who developed brainstem infarction as an initial manifestation of meningovascular syphilis. Similar to our patient, Vaitkus et al.[4] reported the case of a young adult patient with meningovascular neurosyphilis in the form of acute ischaemic stroke presenting with right hemiparesis and speech disturbance. However, their patient was HIV negative. Liu et al.[5] reported that among 149 patients with HIV-negative neurosyphilis, $21(14 \%)$ had ischaemic stroke as the primary symptom (3, syphilitic meningitis; 18, meningovascular neurosyphilis), 15 of whom had ischaemic stroke as the initial manifestation of neurosyphilis. Neurosyphilis is often divided into meningeal, vascular, meningovascular and parenchymatous (including tabes dorsalis and general paresis) forms[1]. The incidence of meningovascular syphilis ranges from $0.3 \%$ to $2.4 \%$ in patients with syphilis[6]. Since the HIV epidemic began, the meningeal and vascular forms of syphilis are more common, and therefore syphilis must be considered in patients with stroke[1].

Characteristic features of syphilis such as cutaneous findings and lymphadenopathy were not evident in our patient, suggesting that central nervous system involvement occurred at a relatively early stage within a few months of infection. Neurosyphilis can occur at any stage of syphilis[3] and meningovascular syphilis usually occurs within 10 years of infection with syphilis[7]. The average latency of meningovascular syphilis is reported to be 7 years; however, meningovascular syphilis can occur within a few months of infection in HIV patients[8], which indicates the difficulty in diagnosing neurosyphilis in patients with HIV.

In our patient, cardiogenic cerebral embolism or atherothrombotic brain infarction was unlikely based on normal cardiac function and carotid sonographic findings. Irregular stenosis observed in the basilar and middle cerebral artery on MR angiography suggested arteritis related to neurosyphilis. The meningovascular form of neurosyphilis is known to show characteristic arteritis: Heubner arteritis and Nissl-Alzheimer arteritis[9]. However, in our patient basilar artery dissection 
could not be fully ruled out since digital subtraction angiography was not performed.

Our patient exhibited occipital pain preceding the onset of neurological deficit. Neurosyphilis may cause prodromal symptoms, such as headache, vertigo, insomnia, emotional lability and personal changes, which can be present for months before the onset of cerebral infarction[3].

In conclusion, acute ischaemic stroke may be the first manifestation of neurosyphilis, especially in young adults. As there is a reported significant misdiagnosis rate of neurosyphilitic ischaemic stroke (81\%)[5], any patient with ischaemic stroke should be screened for neurosyphilis. Furthermore, all patients positive for syphilis should be tested for HIV infection at the time of diagnosis, as it influences the clinical course and treatment options.

\section{Learning Points}

- Acute ischaemic stroke may be the first manifestation of neurosyphilis, especially in young adults.

- Any patient with ischaemic stroke should be screened for neurosyphilis.

- Patients positive for syphilis should be tested for HIV infection at the time of diagnosis.

\section{References}

1. Chahine LM, Khoriaty RN, Tomford WJ, Hussain MS. The changing face of neurosyphilis. Int J Stroke 2011;6:136-143.

2. Bordon J, Martinez-Vazquez C, Alvarez M, Miralles C, Ocampo A, de la Fuente-Aguado J, et al. Neurosyphilis in HIV-infected patients. Eur J Clin Microbiol Infect Dis 1995;14:864-869.

3. Marra CM. Update on neurosyphilis. Curr Infect Dis Rep 2009;11:127-134.

4. Vaitkus A, Krasauskaite E, Urbonaviciute I. Meningovascular neurosyphilis: a report of stroke in a young adult. Medicina (Kaunas) 2010;46:282-285.

5. Liu LL, Zheng $\mathrm{WH}$, Tong ML, Liu GL, Zhang HL, Fu ZG, et al. Ischemic stroke as a primary symptom of neurosyphilis among HIV-negative emergency patients. I Neurol Sci 2012;317:35-39.

6. Gurses C, Bilgic B, Topcular B, Tuncer OG, Akman-Demir G, Hanagasi H, et al. Clinical and magnetic resonance imaging findings of HIV-negative patients with neurosyphilis. J Neurol 2007;254:368-374.

7. Simon RP. Neurosyphilis. Arch Neurol 1985;42:606-613.

8. Johns DR, Tierney M, Felsenstein D. Alteration in the natural history of neurosyphilis by concurrent infection with the human immunodeficiency virus. N Engl J Med 1987;316:15691572.

9. Brightbill TC, Ihmeidan IH, Post MJ, Berger JR, Katz DA. Neurosyphilis in HIV-positive and HIV-negative patients: neuroimaging findings. AJNR Am J Neuroradiol 1995;16:703-711. 